\title{
Retraction
}

\section{Retracted: An Inverse Spectral Problem for the Sturm-Liouville Operator on a Three-Star Graph}

\author{
International Scholarly Research Notices \\ Received 9 July 2014; Accepted 9 July 2014; Published 29 October 2014 \\ Copyright (C) 2014 International Scholarly Research Notices. This is an open access article distributed under the Creative Commons \\ Attribution License, which permits unrestricted use, distribution, and reproduction in any medium, provided the original work is \\ properly cited.
}

This paper [1] has been retracted as it is essentially identical in content with the published article "Determination of SturmLiouville operator on a three-star graph from four spectra," by Dehghani Tazehkand and Akbarfam, published in Acta Universitatis Apulensis No. 32/2012, pp. 147-172.

\section{References}

[1] I. Dehghani Tazehkand and A. J. Akbarfam, "An inverse spectral problem for the Sturm-Liouville operator on a three-star graph," ISRN Applied Mathematics, vol. 2012, Article ID 132842, 23 pages, 2012. 


\section{An Inverse Spectral Problem for the Sturm-Liouville Operator on a Three-Star Graph}

\section{Dehghani Tazehkand and A. Jodayree Akbarfam}

Faculty of Mathematical Sciences, University of Tabriz, 29 Bahman Boulevard, Tabriz, Iran

Correspondence should be addressed to I. Dehghani Tazehkand, isadehghani@gmail.com

Received 10 January 2012; Accepted 15 March 2012

Academic Editor: D. Georges

Copyright (c) 2012 I. Dehghani Tazehkand and A. Jodayree Akbarfam. This is an open access article distributed under the Creative Commons Attribution License, which permits unrestricted use, distribution, and reproduction in any medium, provided the original work is properly cited.

We study an inverse spectral problem for the Sturm-Liouville operator on a three-star graph with the Dirichlet and Robin boundary conditions in the boundary vertices and matching conditions in the internal vertex. As spectral characteristics, we consider the spectrum of the main problem together with the spectra of two Dirichlet-Dirichlet problems and one Robin-Dirichlet problem on the edges of the graph and investigate their properties and asymptotic behavior. We prove that if these four spectra do not intersect, then the inverse problem of recovering the operator is uniquely solvable. We give an algorithm for the solution of the inverse problem with respect to this quadruple of spectra.

\section{Introduction}

This paper is devoted to the study of the inverse spectral problem for Sturm-Liouville operators on a three-star graph with the Dirichlet and Robin boundary conditions in the boundary vertices and matching conditions in the internal vertex. The considered inverse problem consists of recovering the Sturm-Liouville operator on a graph from the given spectral characteristics. Differential operators on graphs (networks, trees) often appear in mathematics, mechanics, physics, geophysics, physical chemistry, electronics, nanoscale technology and branches of natural sciences and engineering (see [1-7] and the bibliographies thereof). In recent years there has been considerable interest in the spectral theory of Sturm-Liouville operators on graphs (see [8-10]). The direct spectral and scattering problems on compact and noncompact graphs, respectively, were considered in many publications (see, e.g., [11-15]). The considered inverse spectral problem is not studied yet. However, inverse spectral problems of recovering differential operators on star-type graphs with the boundary 
conditions other than considered here were studied in $[16,17]$ and other papers. HochstadtLiberman type inverse problems on star-type graphs were investigated in $[16,18]$.

We consider a three-star graph $G$ with vertex set $V=\left\{v_{0}, v_{1}, v_{2}, v_{3}\right\}$ and edge set $E=$ $\left\{e_{1}, e_{2}, e_{3}\right\}$, where $v_{1}, v_{2}, v_{3}$ are the boundary vertices, $v_{0}$ is the internal vertex, and $e_{j}=\left[v_{j}, v_{0}\right]$ for $j=1,2,3$. We assume that the length of every edge is equal to $a, a>0$. Every edge $e_{j} \in E$ is viewed as an interval $[0, a]$. Parametrizing $e_{j} \in E$ by $x \in[0, a]$, the following choice of orientation is convenient for us: $x=0$ corresponds to the boundary vertices $v_{1}, v_{2}, v_{3}$ and $x=a$ corresponds to the internal vertex $v_{0}$. A function $Y$ on $G$ may be represented as a vector $Y(x)=\left[y_{j}(x)\right]_{j=1,2,3}, x \in[0, a]$ and the function $y_{j}(x)$ is defined on the edge $e_{j}$. Let $q(x)=\left[q_{j}(x)\right]_{j=1,2,3}$ be a function on $G$ which is called the potential and $q_{j}(x) \in L_{2}(0, a)$ is a real-valued function defined on the edge $e_{j}$. Let us consider the following Sturm-Liouville equations on $G$ :

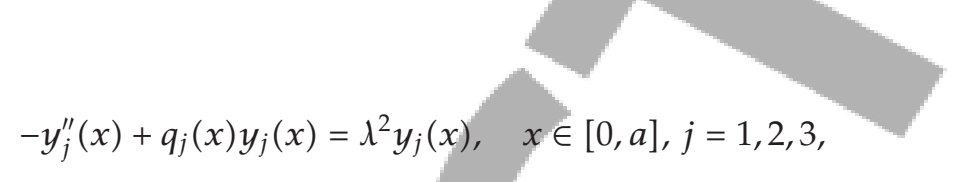

where $\lambda$ is the spectral parameter. The functions $y_{j}(x)$ and $y_{j}^{\prime}(x)$ are absolutely continuous and satisfy the following matching conditions in the internal vertex $v_{0}$ :

$$
\begin{gathered}
y_{i}(a)=y_{j}(a) \text { for } i, j=1,2,3,(\text { continuity condition), } \\
\sum_{j=1}^{3} y_{j}^{\prime}(a)+\beta y_{1}(a, \lambda)=0 \quad \text { (Kirchhoff's condition), }
\end{gathered}
$$

where $\beta$ is a real number. In electrical circuits, (1.2) expresses Kirchhof's law; in an elastic string network, it expresses the balance of tension and so on. Let us denote by $L_{0}$ the boundary-value problem for (1.1) with the matching conditions (1.2) and the following boundary conditions at the boundary vertices $v_{1}, v_{2}, v_{3}$ :

where $h$ is a real number.

$$
y_{1}(0)=y_{2}(0)=y_{3}^{\prime}(0)-h y_{3}(0)=0
$$

The problem of small transverse vibrations of a three-star graph consisting of three inhomogeneous smooth strings joined at the internal vertex with two pendent ends fixed and one pendent end which can move without friction in the directions orthogonal to their respective equilibrium positions can be reduced to this problem by the Liouville transformation. This problem occurs also in quantum mechanics when one considers a quantum particle subject to the Shrödinger equation moving in a quasi-one-dimensional graph domain.

In this paper, we study the inverse problem of recovering the potential $q(x)=$ $\left[q_{j}(x)\right]_{j=1,2,3}$ and the real numbers $h$ and $\beta$ from the given spectral characteristics. Similar inverse spectral problems on star-type graphs with three and arbitrary number of edges but only with the Dirichlet conditions at the boundary vertices were considered in $[16,17]$. As spectral characteristics, we consider the set of eigenvalues of problem $L_{0}$ together with 
the sets of eigenvalues of the following two Dirichlet-Dirichlet problems and one RobinDirichlet problem on the edges of the graph G:

$$
\begin{gathered}
-y_{j}^{\prime \prime}(x)+q_{j}(x) y_{j}(x)=\lambda^{2} y_{j}(x), \quad x \in[0, a], \\
y_{j}(0)=y_{j}(a)=0, \quad j=1,2, \\
-y_{3}^{\prime \prime}(x)+q_{3}(x) y_{3}(x)=\lambda^{2} y_{3}(x), \quad x \in[0, a], \\
y_{3}^{\prime}(0)-h y_{3}(0)=y_{3}(a)=0,
\end{gathered}
$$

through which we denote these problems by $L_{j}, j=1,2,3$. We obtain conditions for four sequences of real numbers that enable one to reconstruct the potential $q(x)=\left[q_{j}(x)\right]_{j=1,2,3}$ and the real numbers $h$ and $\beta$ so that one of the sequences describes the spectrum of the boundaryvalue problem $L_{0}$ and other three sequences coincide with the spectra of the problems $L_{j}, j=$ $1,2,3$. We give an algorithm for the construction of the potential and the coefficients of the boundary and matching conditions corresponding to these four sequences.

Denote by $L_{j}^{\prime}, j=1,2,3$ the following boundary-value problems:

$$
\begin{gathered}
-y_{j}^{\prime \prime}(x)(x)+q_{j}(x) y_{j}(x)=\lambda^{2} y_{j}(x), \quad x \in[0, a], \\
y_{j}(0)=y_{j}^{\prime}(a)=0, \quad j=1,2, \\
-y_{3}^{\prime \prime}(x)+q_{3}(x) y_{3}(x)=\lambda^{2} y_{3}(x), \quad x \in[0, a], \\
y_{3}^{\prime}(0)-h y_{3}(0)=y_{3}^{\prime}(a)=0 .
\end{gathered}
$$

The main idea of the solution of the inverse problem for the considered system is its reduction to three independent inverse problems of reconstruction of the functions $q_{j}(x) \in L_{2}(0, a), j=$ $1,2,3$ and $h$ on the basis of two spectra, namely, the spectrum of the problem $L_{j}$ and the spectrum of the problem $L_{j}^{\prime}$. Since the solutions of the later inverse problems are known (see $[19$, Section 1.5], [20, Section 3.4]), this reduction gives an algorithm for the reconstruction of the potential and coefficients of the boundary-value problem $L_{0}$.

Let us consider the operator-theoretical interpretation of our problem. Denote by A the operator acting in the Hilbert space $H=L_{2}(0, a) \oplus L_{2}(0, a) \oplus L_{2}(0, a)$ with standard inner product $(\cdot, \cdot)_{H}$, according to the formulas

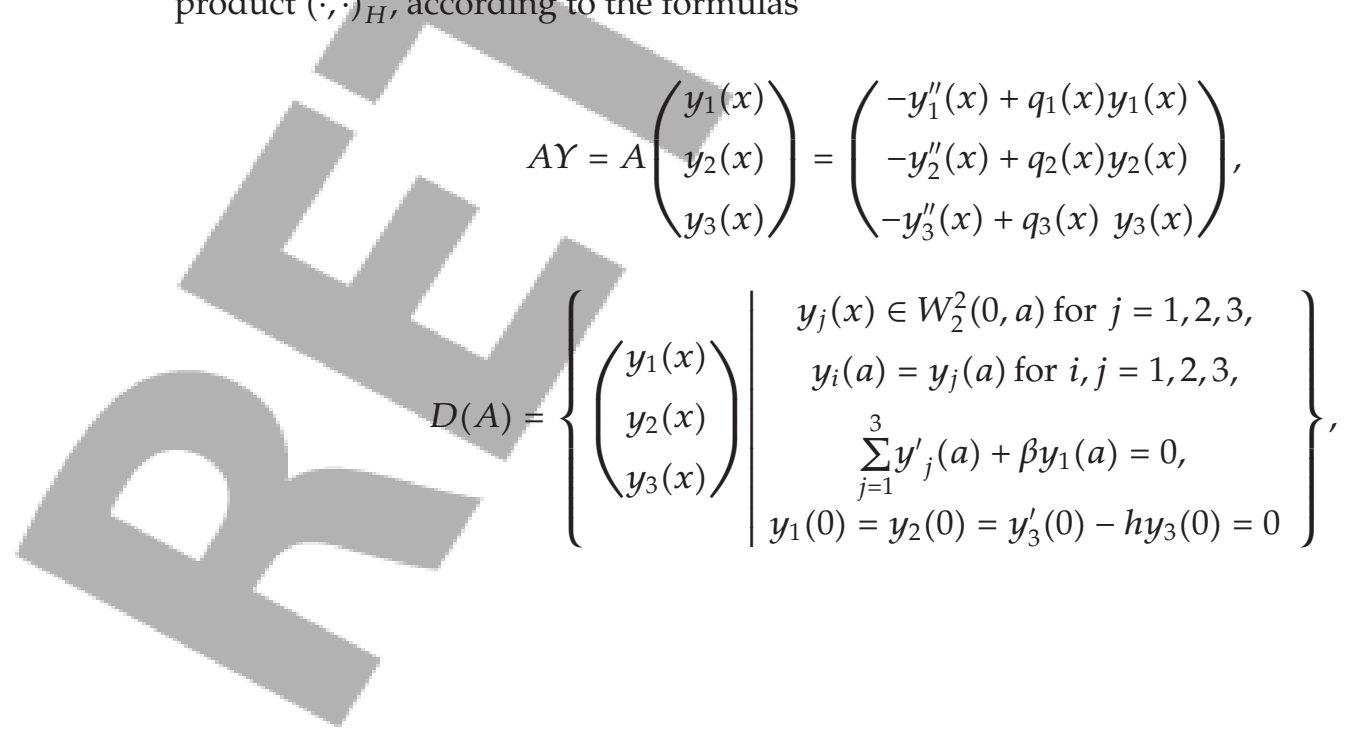


where $W_{2}^{2}(0, a)$ is a Sobolev space. By constructing the adjoint operator $A^{*}$, it is easy to show that $A$ is self-adjoint. The operator $A$ has a discrete spectrum and its eigenvalues coincide with the squares of the eigenvalues of the boundary-value problem $L_{0}$. Thus, for all eigenvalues of the boundary-value problem $L_{0}$ to be real and nonzero, it is necessary and sufficient that the operator $A$ be strictly positive $(A \gg 0)$. Furthermore, integrating by parts, we obtain the following equality for any vector function $Y=\left(y_{1}(x), y_{2}(x), y_{3}(x)\right)^{t} \in D(A)(t$ denotes the transpose of a matrix):

$$
(A Y, Y)_{H}=\sum_{j=1}^{3} \int_{0}^{a}\left(\left|y_{j}^{\prime}(x)\right|^{2}+q_{j}(x)\left|y_{j}(x)\right|^{2}\right) d x+\beta\left|y_{1}(a)\right|^{2}+h\left|y_{3}(0)\right|^{2} .
$$

Relation (1.7) yields the following simple sufficient condition for the strict positivity of the operator $A$ :

$$
q_{j}(x) \geq \epsilon>0 \quad \text { a.e. on }[0, a], j=1,2,3, \beta \geq 0, h \geq 0 .
$$

On the other hand, if $A \gg 0$, then setting in turn $Y=\left(y_{1}(x), 0,0\right)^{t} \in D(A), Y=\left(0, y_{2}(x), 0\right)^{t} \in$ $D(A)$ and $Y=\left(0,0, y_{3}(x)\right)^{t} \in D(A)$ in (1.7), we establish that the eigenvalues of the problems $L_{j}, j=1,2,3$ are also real and nonzero. The strict positivity of the operator $A$ can be realized by shifting the spectral parameter $\lambda^{2}-q_{0}, q_{0}>0$, in (1.1). For this reason, we assume in what follows without loss of generality that $A \gg 0$. Thus, the eigenvalues of the boundary-value problems $L_{0}$ and $L_{j}, j=1,2,3$ are nonzero real numbers.

This paper has the following structure: in Section 2 the direct problem is considered. Aspects of the theory of entire and meromorphic functions are used as tools for a description of the set of eigenvalues of the boundary-value problem $L_{0}$ and the spectra of the auxiliary problems $L_{j}, j=1,2,3$ associated with this system. As a consequence we prove that the eigenvalues of the main problem and the spectra of the auxiliary problems interlace in some sense. In Section 3 we solve the inverse spectral problem for $L_{0}$ within the framework of the statement indicated above.

\section{Direct Problem}

In this section, we describe the properties of sequences of eigenvalues of the boundary-value problems $L_{0}$ and $L_{j}, j=1,2,3$ that are necessary for what follows.

Let us denote by $c_{j}(x, \lambda), s_{j}(x, \lambda), j=1,2,3$ the solutions of (1.1) on the edge $e_{j}$ which satisfy the initial conditions

$$
c^{\prime}{ }_{j}(0, \lambda)=c_{j}(0, \lambda)-1=0, \quad s_{j}(0, \lambda)=s_{j}^{\prime}(0, \lambda)-1=0 .
$$

For each fixed $x \in[0, a]$, the functions $c_{j}^{(v)}(x, \lambda)$ and $s_{j}^{(v)}(x, \lambda), v=0,1, j=1,2,3$ are entire in $\lambda$. Since $\left\{c_{j}(x, \lambda), s_{j}(x, \lambda)\right\}$ is a fundamental system of solutions of (1.1) on the edge $e_{j}$, then the solutions of (1.1), which satisfy the conditions (1.3), are

$$
y_{j}(x, \lambda)=C_{j} u_{j}(x, \lambda), \quad j=1,2,3,
$$


where $C_{j}, j=1,2,3$ are constants and

$$
u_{j}(x, \lambda)= \begin{cases}s_{j}(x, \lambda), & j=1,2 \\ c_{3}(x, \lambda)+h s_{3}(x, \lambda), & j=3 .\end{cases}
$$

Substituting (2.2) into (1.2), we establish that the eigenvalues of the boundary-value problem $L_{0}$ are zeros of the entire function

or

$$
\Phi(\lambda):=\left|\begin{array}{ccc}
u_{1}(a, \lambda) & -u_{2}(a, \lambda) & 0 \\
u_{1}(a, \lambda) & 0 & -u_{3}(a, \lambda) \\
u_{1}^{\prime}(a, \lambda)+\beta u_{1}(a, \lambda) & u_{2}^{\prime}(a, \lambda) & u_{3}^{\prime}(a, \lambda)
\end{array}\right|
$$

$$
\Phi(\lambda)=\sum_{i=1}^{3}\left(u_{i}^{\prime}(a, \lambda) \prod_{\substack{j=1 \\ j \neq i}}^{3} u_{j}(a, \lambda)\right)+\beta \prod_{j=1}^{3} u_{j}(a, \lambda)
$$

For what follows, we need the definition presented below.

Definition 2.1 (see [21]). Let $\left\{z_{k}\right\}_{-\infty}^{\infty}\left(\left\{z_{k}\right\}_{-\infty, k \neq 0}^{\infty}\right)$ be a sequence of complex numbers of finite multiplicities which satisfy the following conditions: (1) the sequence is symmetric with respect to the imaginary axis and symmetrically located numbers possess the same multiplicities; (2) any strip $|\operatorname{Re} z| \leq p<\infty$ contains not more than a finite number of $z_{k}$. Then, the following way of enumeration is called proper:

(i) $z_{-k}=-\overline{z_{k}}\left(\operatorname{Re} z_{k} \neq 0\right)$;

(ii) $\operatorname{Re} z_{k} \leq \operatorname{Re} z_{k+1}$;

(iii) the multiplicities are taken into account.

If a sequence has even number of pure imaginary elements, we exclude the index zero from enumeration to make it proper.

Throughout Section 2, denote

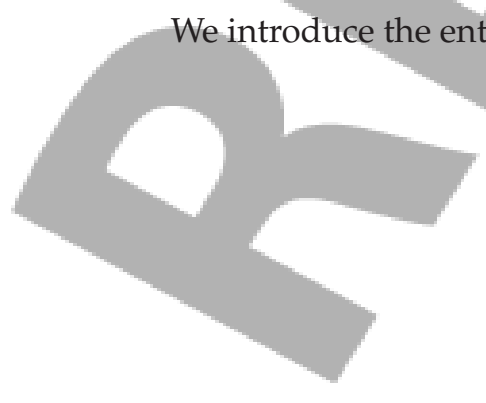

$$
B_{j}= \begin{cases}\frac{1}{2} \int_{0}^{a} q_{j}(x) d x, & j=1,2, \\ h+\frac{1}{2} \int_{0}^{a} q_{3}(x) d x, & j=3 .\end{cases}
$$

$$
\Psi(\lambda)=\prod_{j=1}^{3} u_{j}(a, \lambda)
$$


Let us denote by $\left\{\lambda_{k}\right\}_{-\infty, k \neq 0}^{\infty}$ the set of zeros of $\Phi(\lambda)$ and by $\left\{\kappa_{k}\right\}_{-\infty, k \neq 0}^{\infty}$ the set of zeros of the function $\Psi(\lambda)$. Denote by $\left\{v_{k}^{(j)}\right\}_{-\infty, k \neq 0}^{\infty}, j=1,2,3$ the sets of zeros of the functions $u_{j}(a, \lambda), j=1,2,3$, respectively. It is clear from (2.7) that the set $\left\{\kappa_{k}\right\}_{-\infty, k \neq 0}^{\infty}$ is the union of the sets $\bigcup_{j=1}^{3}\left\{v_{k}^{(j)}\right\}_{-\infty, k \neq 0}^{\infty}$, that is, the spectra of the auxiliary problems $L_{j}, j=1,2,3$. According to the remark presented in Section 1, all numbers $\lambda_{k}, v_{k}^{(j)}, j=1,2,3$ and $\kappa_{k}$ are real and nonzero. We enumerate the sets $\left\{\lambda_{k}\right\}_{-\infty, k \neq 0}^{\infty},\left\{v_{k}^{(j)}\right\}_{-\infty, k \neq 0}^{\infty}, j=1,2,3$ and $\left\{\kappa_{k}\right\}_{-\infty, k \neq 0}^{\infty}$ in the proper way $\left(\lambda_{-k}=-\lambda_{k}, \lambda_{k} \leq \lambda_{k+1}, v_{-k}^{(j)}=-v_{k}^{(j)}, v_{k}^{(j)}<v_{k+1}^{(j)}\right.$ for $j=1,2,3$ and $\left.\kappa_{-k}=-\kappa_{k}, \kappa_{k} \leq \kappa_{k+1}\right)$. Note that the sets of eigenvalues $\left\{v_{k}^{(j)}\right\}_{-\infty, k \neq 0}^{\infty}, j=1,2,3$ behave asymptotically as follows (see [20, Section 1.5]):

$$
\begin{aligned}
& v_{k}^{(j)}=\frac{k \pi}{a}+\frac{B_{j}}{\pi k}+\frac{\delta_{k}^{(j)}}{k}, j=1,2 \\
& v_{k}^{(3)}=\frac{\pi(k-(1 / 2))}{a}+\frac{B_{3}}{\pi(k-(1 / 2))}+\frac{\delta_{k}^{(3)}}{k}
\end{aligned}
$$

where $\left\{\delta_{k}^{(j)}\right\}_{-\infty k \neq 0}^{\infty} \in l_{2}$ for $j=1,2,3$.

Let us denote by $L^{d}, d>0$ the class (introduced in [22, page 149]) of entire functions of exponential type $\leq d$ whose restrictions on the real line belong to $L_{2}(-\infty, \infty)$.

Lemma 2.2. The functions $\Phi(\lambda)$ and $\Psi(\lambda)$ can be represented as follows:

$$
\begin{aligned}
\Phi(\lambda)= & \frac{2 \sin \lambda a-3 \sin ^{3} \lambda a}{\lambda}+\left(2 B_{1}+2 B_{2}+3 B_{3}+\beta\right) \frac{\sin ^{2} \lambda a \cos \lambda a}{\lambda^{2}} \\
& -\left(B_{1}+B_{2}\right) \frac{\cos ^{3} \lambda a}{\lambda^{2}}+\frac{\omega_{1}(\lambda)}{\lambda^{2}} \\
\Psi(\lambda)= & \frac{\sin ^{2} \lambda a \cos \lambda a}{\lambda^{2}}-\left(B_{1}+B_{2}\right) \frac{\cos ^{2} \lambda a \sin \lambda a}{\lambda^{3}}+B_{3} \frac{\sin ^{3} \lambda a}{\lambda^{3}}+\frac{\omega_{2}(\lambda)}{\lambda^{3}},
\end{aligned}
$$

where $\omega_{1}(\lambda), \omega_{2}(\lambda) \in L^{3 a}$.

Proof. Using the formulas of [19, page 18], [20, page 9] and taking into account that

$$
\int_{0}^{a} f(t) \cos \lambda t d t \in L^{a}, \quad \int_{0}^{a} f(t) \sin \lambda t d t \in L^{a}
$$

whenever $f \in L_{2}(0, a)$ by the Paley-Wiener theorem [23, page 103], we obtain

$$
\begin{aligned}
& u_{j}(a, \lambda)=\frac{\sin \lambda a}{\lambda}+\frac{\rho_{j 1}(\lambda)}{\lambda}=\frac{\sin \lambda a}{\lambda}-B_{j} \frac{\cos \lambda a}{\lambda^{2}}+\frac{\rho_{j 2}(\lambda)}{\lambda^{2}}, \quad j=1,2, \\
& u_{3}(a, \lambda)=\cos \lambda a+\varrho_{31}(\lambda)=\cos \lambda a+B_{3} \frac{\sin \lambda a}{\lambda}+\frac{\varrho_{32}(\lambda)}{\lambda},
\end{aligned}
$$


ISRN Applied Mathematics

$$
\begin{aligned}
& u_{j}^{\prime}(a, \lambda)=\cos \lambda a+B_{j} \frac{\sin \lambda a}{\lambda}+\frac{\sigma_{j}(\lambda)}{\lambda}, \quad j=1,2, \\
& u_{3}^{\prime}(a, \lambda)=-\lambda \sin \lambda a+B_{3} \cos \lambda a+\sigma_{3}(\lambda),
\end{aligned}
$$

where $\varrho_{j 1}(\lambda), \varrho_{j 2}(\lambda), \sigma_{j}(\lambda), j=1,2,3$, are entire functions of class $L^{a}$. Substituting (2.12) into (2.5) and (2.7), we get (2.9) and (2.10).

Theorem 2.3. The set $\left\{\lambda_{k}\right\}_{-\infty, k \neq 0}^{\infty}$ of zeros of $\Phi(\lambda)$ can be represented as the union of three pairwise disjoint subsequences $\bigcup_{j=1}^{3}\left\{\lambda_{k}^{(j)}\right\}_{-\infty, k \neq 0}^{\infty}$ which, being enumerated in the following way: $\lambda_{-k}^{(1)}=-\lambda_{k}^{(1)}$, $\lambda_{-k}^{(2)}=-\lambda_{k}^{(3)}, \lambda_{-k}^{(3)}=-\lambda_{k}^{(2)}$, and $\lambda_{k}^{(j)} \leq \lambda_{k+1}^{(j)}$ for $j=1,2,3$, behave asymptotically as follows:

$$
\begin{gathered}
\lambda_{k}^{(1)}=\frac{k \pi}{a}+\frac{B_{1}+B_{2}}{2 k \pi}+\frac{r_{k}^{(1)}}{k}, \\
\lambda_{k}^{(j)}=\frac{k \pi+(-1)^{j} \sin ^{-1} \sqrt{2 / 3}}{a}+\frac{3 B_{1}+3 B_{2}+6 B_{3}+2 \beta}{12 k \pi}+\frac{r_{k}^{(j)}}{k}, j=2,3,
\end{gathered}
$$

where $\left\{\gamma_{k}^{(j)}\right\}_{-\infty, k \neq 0}^{\infty} \in l_{2}$ for $j=1,2,3$

Proof. In the same way as [16, Lemma 1.3], we can show that the set of zeros $\left\{\lambda_{k}\right\}_{-\infty, k \neq 0}^{\infty}$ can be arranged into three pairwise disjoint subsequences $\left\{\lambda_{k}^{(j)}\right\}_{-\infty, k \neq 0}^{\infty}, j=1,2,3$ enumerated in the following way: $\lambda_{-k}^{(1)}=-\lambda_{k}^{(1)}, \lambda_{-k}^{(2)}=-\lambda_{k}^{(3)}, \lambda_{-k}^{(3)}=-\lambda_{k}^{(2)}$, and $\lambda_{k}^{(j)} \leq \lambda_{k+1}^{(j)}$ for $j=1,2,3$, such that $\left\{\lambda_{k}\right\}_{-\infty, k \neq 0}^{\infty}=\bigcup_{j=1}^{3}\left\{\lambda_{k}^{(j)}\right\}_{-\infty, k \neq 0}^{\infty}$, and

$$
\begin{gathered}
\lambda_{k}^{(1)}=\frac{k \pi}{a}+\varepsilon_{k}^{(1)}, \\
\lambda_{k}^{(j)}=\frac{k \pi+(-1)^{j} \sin ^{-1} \sqrt{2 / 3}}{a}+\varepsilon_{k}^{(j)}, \quad j=2,3,
\end{gathered}
$$

where $\varepsilon_{k}^{(j)}=o(1)$, as $k \rightarrow \infty$ for $j=1,2,3$. It is not difficult to see that

$$
\varepsilon_{k}^{(j)}=O\left(\frac{1}{k}\right), \quad k \longrightarrow \infty, j=1,2,3
$$

Substituting (2.15) into $\lambda_{k}^{(1)} \Phi\left(\lambda_{k}^{(1)}\right)=0$, then from (2.9) and taking into account that the function $\omega_{1}(\lambda)$ is bounded on the real axis by the Paley-Wiener theorem, we obtain

$$
\begin{aligned}
\lambda_{k}^{(1)} \Phi\left(\lambda_{k}^{(1)}\right)= & (-1)^{k}\left(2 \sin \varepsilon_{k}^{(1)} a-3 \sin ^{3} \varepsilon_{k}^{(1)} a\right) \\
& +(-1)^{k} a\left(2 B_{1}+2 B_{2}+3 B_{3}+\beta\right) \frac{\sin ^{2} \varepsilon_{k}^{(1)} a \cos \varepsilon_{k}^{(1)} a}{k \pi}
\end{aligned}
$$




$$
\begin{aligned}
& -(-1)^{k} a\left(B_{1}+B_{2}\right) \frac{\cos ^{3} \varepsilon_{k}^{(1)} a}{k \pi}+\frac{a \omega_{1}\left(\lambda_{k}^{(1)}\right)}{k \pi}+O\left(\frac{1}{k^{2}}\right) \\
= & (-1)^{k} 2 \sin \varepsilon_{k}^{(1)} a+O\left(\frac{1}{k}\right)=0, \quad k \longrightarrow \infty .
\end{aligned}
$$

This yields $\sin \varepsilon_{k}^{(1)} a=O(1 / k)$. Thus, $\varepsilon_{k}^{(1)}=O(1 / k)$. Similarly, we can show that $\varepsilon_{k}^{(j)}=O(1 / k)$ for $j=2$, 3. Substituting (2.15) into the equation $\lambda_{k}^{(1)} \Phi\left(\lambda_{k}^{(1)}\right)=0$, where $\Phi(\lambda)$ is given by (2.9), by expanding the left-hand side of resulting equation in power series and taking into account (2.17) and $\left\{\omega_{1}\left(\lambda_{k}^{(1)}\right)\right\}_{-\infty, k \neq 0}^{\infty} \in l_{2}$ (see [20, Lemma 1.4.3]), we obtain

$$
2 \varepsilon_{k}^{(1)} a-\frac{a\left(B_{1}+B_{2}\right)}{k \pi}+\frac{\tau_{k}}{k}=0
$$

where $\left\{\tau_{k}\right\}_{-\infty, k \neq 0}^{\infty} \in l_{2}$. Solving this equation we get (2.13). In the same way, we get (2.14).

To compare necessary conditions on a sequence to be the spectrum of the boundaryvalue problem $L_{0}$ with the sufficient condition which will be obtained in Section 3, we need more precise asymptotics.

Theorem 2.4. Let $q_{j}(x) \in W_{2}^{1}(0, a)$ for $j=1,2,3$. Then the subsequences of Theorem 2.3 behave asymptotically as follows:

$$
\begin{gathered}
\lambda_{k}^{(1)}=\frac{k \pi}{a}+\frac{B_{1}+B_{2}}{2 k \pi}+\frac{r_{k}^{(1)}}{k^{2}} \\
\lambda_{k}^{(j)}=\frac{k \pi+(-1)^{j} \sin ^{-1} \sqrt{2 / 3}}{a}+\frac{3 B_{1}+3 B_{2}+6 B_{3}+2 \beta}{12 k \pi}+\frac{r_{k}^{(j)}}{k^{2}}, \quad j=2,3,
\end{gathered}
$$

where $\left\{\gamma_{k}^{(j)}\right\}_{-\infty, k \neq 0}^{\infty} \in l_{2}$ for $j=1,2,3$.

Proof. If $q_{j}(x) \in W_{2}^{1}(0, a)$, twice integrating by parts the formulas of [20, page 9] and [19, page 18], we obtain

$$
\begin{aligned}
& u_{j}(a, \lambda)=\frac{\sin \lambda a}{\lambda}-B_{j} \frac{\cos \lambda a}{\lambda^{2}}+D_{j} \frac{\sin \lambda a}{\lambda^{3}}+\frac{\rho_{j}(\lambda)}{\lambda^{3}}, \quad j=1,2, \\
& u_{3}(a, \lambda)=\cos \lambda a+B_{3} \frac{\sin \lambda a}{\lambda}+D_{3} \frac{\cos \lambda a}{\lambda^{2}}+\frac{\rho_{3}(\lambda)}{\lambda^{2}}, \\
& u_{j}^{\prime}(a, \lambda)=\cos \lambda a+B_{j} \frac{\sin \lambda a}{\lambda}+D_{j}^{\prime} \frac{\cos \lambda a}{\lambda^{2}}+\frac{\sigma_{j}(\lambda)}{\lambda^{2}}, \quad j=1,2, \\
& u_{3}^{\prime}(a, \lambda)=-\lambda \sin \lambda a+B_{3} \cos \lambda a+D_{3}^{\prime} \frac{\sin \lambda a}{\lambda}+\frac{\sigma_{3}(\lambda)}{\lambda}
\end{aligned}
$$


where $D_{j}, D_{j}^{\prime}, j=1,2,3$ are constants and $\rho_{j}(\lambda), \sigma_{j}(\lambda), j=1,2,3$ are entire functions of class $L^{a}$. Substituting (2.22) into (2.5), we obtain

$$
\begin{aligned}
\Phi(\lambda)= & \frac{2 \sin \lambda a-3 \sin ^{3} \lambda a}{\lambda}+\left(2 B_{1}+2 B_{2}+3 B_{3}+\beta\right) \frac{\sin ^{2} \lambda a \cos \lambda a}{\lambda^{2}} \\
& -\left(B_{1}+B_{2}\right) \frac{\cos ^{3} \lambda a}{\lambda^{2}}+E_{1} \frac{\sin ^{3} \lambda a}{\lambda^{3}}+E_{2} \frac{\cos ^{2} \lambda a \sin \lambda a}{\lambda^{3}}+\frac{\omega_{3}(\lambda)}{\lambda^{3}}
\end{aligned}
$$

where $E_{1}, E_{2}$ are constants and $\omega_{3}(\lambda) \in L^{3 a}$. Substituting (2.13) into the equation $\lambda_{k}^{(1)} \Phi\left(\lambda_{k}^{(1)}\right)=$ 0 , where $\Phi(\lambda)$ is given by (2.23) and by expanding the left-hand side of resulting equation in power series, we get (2.20). Analogously, we obtain (2.21). Theorem 2.4 is proved.

Remark 2.5. Under the conditions of Theorem 2.4, the spectra $\left\{v_{k}^{(j)}\right\}_{-\infty, k \neq 0}^{\infty}$ of the boundaryvalue problems $L_{j}$ for $j=1,2,3$ behave asymptotically as follows (see [20, page 75]):

$$
\begin{aligned}
& v_{k}^{(j)}=\frac{k \pi}{a}+\frac{B_{j}}{\pi k}+\frac{\delta_{k}^{(j)}}{k^{2}}, \quad j=1,2, \\
& v_{k}^{(3)}=\frac{\pi(k-(1 / 2))}{a}+\frac{B_{3}}{\pi(k-(1 / 2))}+\frac{\delta_{k}^{(3)}}{k^{2}},
\end{aligned}
$$

where $\left\{\delta_{k}^{(j)}\right\}_{-\infty, k \neq 0}^{\infty} \in l_{2}$ for $j=1,2,3$.

For investigation of direct and inverse spectral problems, methods of the theory of entire and meromorphic functions are widely used. For this reason, we give several notation and definitions for what follows.

If $\Omega \subseteq \mathbb{C}$ is an open set, we denote by $\mathscr{\ell}(\Omega)$ the set of all functions which are analytic in $\Omega$ and by $\mathcal{M}(\Omega)$ the set of all functions meromorphic in $\Omega$.

Definition 2.6 (see [24]). Let $\mathcal{K} \subseteq \mathcal{M}(\mathbb{C})$ and let $\varphi, \psi \in \mathscr{H}(\mathbb{C})$.

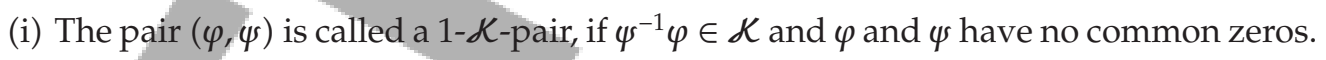

(ii) Let $n \in \mathbb{N}$ and $n \geq 2$. The pair $(\varphi, \psi)$ is called an $n$ - $\mathcal{K}$-pair, if $\psi^{-1} \varphi \in \mathcal{K}$, and there exist 1- $\mathcal{K}$-pairs $\left(\varphi_{1}, \psi_{1}\right), \ldots,\left(\varphi_{n}, \psi_{n}\right)$ such that

and no representation of this kind is possible with less than $n$ many $1-\mathcal{K}$-pairs.

Definition 2.7 (see [24]). A function $f \in \mathscr{H}(\mathbb{C} \backslash \mathbb{R})$ is said to be of Nevanlinna class $\mathcal{N}$ if

(i) $f(z)=\overline{f(\bar{z})}$ for $z \in \mathbb{C} \backslash \mathbb{R}$;

(ii) $\operatorname{Im} f(z) \geq 0$ for $\operatorname{Im} z>0$. 
Definition 2.8 (see [24]). The class $\mathcal{N}^{\mathrm{eP}}$ of essentially positive Nenalinna functions is defined as the set of all functions $f \in \mathcal{N}$ which are analytic in $\mathbb{C} \backslash[0, \infty)$ with possible exception of finitely many poles. Moreover, the class $\mathcal{N}_{-}^{\text {ep }}$ is defined as the set of all functions $f \in \mathcal{N}$ such that for some $b \in \mathbb{R}$ we have $f \in \mathscr{H}(\mathbb{C} \backslash[b, \infty))$ and $f(z) \leq 0$ for $z \in(-\infty, b)$.

It is easy to check that $\mathcal{N}_{-}^{\mathrm{ep}} \subseteq \mathcal{N}^{\mathrm{ep}}$.

Definition 2.9 (see [25]). An entire function $\omega(z)$ of exponential type $\sigma>0$ is said to be a function of sine-type if it satisfies the following conditions:

(i) all the zeros of $\omega(z)$ lie in a strip $|\operatorname{Im} z|<h<\infty$;

(ii) for some $h_{1}$ and all $z \in\left\{\lambda: \operatorname{Im} z=h_{1}\right\}$, the following inequalities hold:

$$
0<m \leq|\omega(z)| \leq M<\infty
$$

(iii) the type of $\omega(z)$ in the lower half-plane coincides with that in the upper half-plane.

Let us introduce the entire functions

$$
\begin{gathered}
\varphi_{j}(z)=-u_{j}^{\prime}(a, \sqrt{z})-\frac{\beta}{3} u_{j}(a, \sqrt{z}), \quad j=1,2,3, \\
\psi_{j}(z)=u_{j}(a, \sqrt{z}), \quad j=1,2,3, \\
\varphi(z)=-\Phi(\sqrt{z}), \quad \psi(z)=\Psi(\sqrt{z}) .
\end{gathered}
$$

Using (2.5) and (2.7), we obtain

and consequently

$$
\varphi(z)=\sum_{i=1}^{3}\left(\varphi_{i}(z) \prod_{\substack{j=1 \\ j \neq i}}^{3} \psi_{j}(z)\right), \quad \psi(z)=\prod_{j=1}^{3} \psi_{j}(z)
$$

Lemma 2.10. (1) the zeros of the functions $\varphi_{j}(z)$ and $\psi_{j}(z)(j=1,2,3)$ are real;

(2) the functions $\varphi_{j}(z)$ and $\psi_{j}(z)(j=1,2,3)$ have no common zeros.

Proof. The zeros of $\varphi_{j}(z), j=1,2,3$ coincide with the squares of the eigenvalues of the boundary-value problems

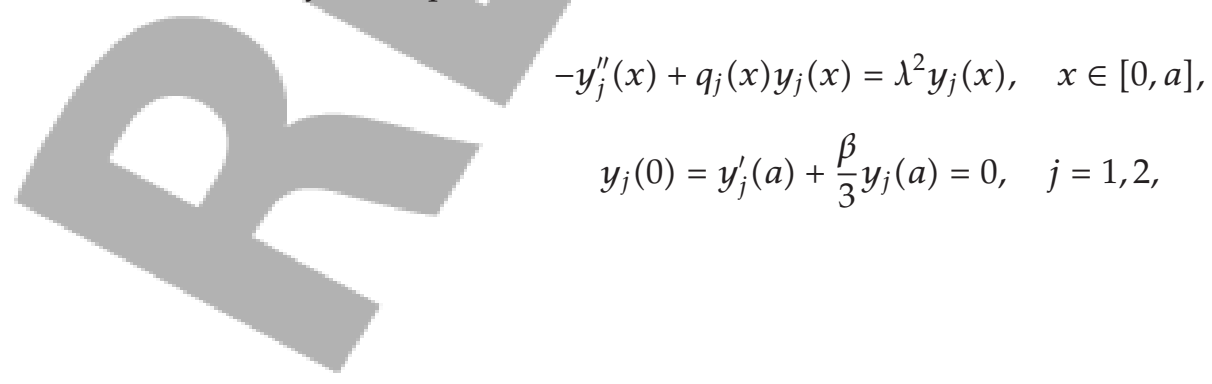


ISRN Applied Mathematics

$$
\begin{gathered}
-y_{3}^{\prime \prime}(x)+q_{3}(x) y_{3}(x)=\lambda^{2} y_{3}(x), \quad x \in[0, a], \\
y_{3}^{\prime}(0)-h y_{3}(0)=y_{3}^{\prime}(a)+\frac{\beta}{3} y_{3}(a)=0,
\end{gathered}
$$

respectively, and the zeros of $\psi_{j}(z)$ coincide with the squares of the eigenvalues of the boundary-value problems $L_{j}, j=1,2,3$, respectively. These problems are self-adjoint and it follows from [26, Part I, Theorem 3] that the squares of their eigenvalues are real. Assertion 1 is proved. To prove assertion 2, let $z_{0}$ be a common zero of $\varphi_{j}(z)$ and $\psi_{j}(z)$. Using the Lagrange identity(see [26, Part II, page 50]) for solutions $u_{j}(a, \sqrt{z})$ and $u_{j}\left(a, \sqrt{z_{0}}\right)$ of (1.1), we obtain

$$
\begin{aligned}
\left(z-z_{0}\right) \int_{0}^{a} u_{j}(x, \sqrt{z}) u_{j}\left(x, \sqrt{z_{0}}\right) d x & =\left.\left(u_{j}(x, \sqrt{z}) u_{j}^{\prime}\left(x, \sqrt{z_{0}}\right)-u_{j}^{\prime}(x, \sqrt{z}) u_{j}\left(x, \sqrt{z_{0}}\right)\right)\right|_{0} ^{a} \\
& =\varphi_{j}(z) \psi_{j}\left(z_{0}\right)-\varphi_{j}\left(z_{0}\right) \psi_{j}(z) .
\end{aligned}
$$

For $z \rightarrow z_{0}$ we get

$$
\int_{0}^{a} u_{j}^{2}\left(x, \sqrt{z_{0}}\right) d x=\dot{\varphi}_{j}\left(z_{0}\right) \psi_{j}\left(z_{0}\right)-\varphi_{j}\left(z_{0}\right) \dot{\varphi}_{j}\left(z_{0}\right)=0,
$$

where $\dot{\varphi}_{j}(z)=(d / d z) \varphi_{j}(z)$ and $\dot{\psi}_{j}(z)=(d / d z) \psi_{j}(z)$. This implies that $u_{j}\left(x, \sqrt{z_{0}}\right) \equiv 0$ which is a contradiction. Therefore, $\varphi_{j}(z)$ and $\psi_{j}(z)$ have no common zeros.

Lemma 2.11. The functions $\varphi_{j}(z) / \psi_{j}(z), j=1,2,3$ and $\varphi(z) / \psi(z)$ are of the Nevanlinna class $\mathcal{N}$.

Proof. Let $j \in\{1,2,3\}$. Using the Lagrange identity for the solution $u_{j}(a, \sqrt{z})$ of $(1.1)$, we have

$$
\left.\left(\overline{u_{j}^{\prime}(x, \sqrt{z})} u_{j}(x, \sqrt{z})-u_{j}^{\prime}(x, \sqrt{z}) \overline{u_{j}(x, \sqrt{z})}\right)\right|_{0} ^{a}=2 i \operatorname{Im} z \int_{0}^{a}\left|u_{j}(x, \sqrt{z})\right|^{2} d x .
$$

Since

$$
\operatorname{Im}\left(\overline{u_{j}^{\prime}(a, \sqrt{z})} u_{j}(a, \sqrt{z})-u_{j}^{\prime}(a, \sqrt{z}) \overline{u_{j}(a, \sqrt{z})}\right)=-2\left|u_{j}(a, \sqrt{z})\right|^{2} \operatorname{Im} \frac{u_{j}^{\prime}(a, \sqrt{z})}{u_{j}(a, \sqrt{z})}
$$

then (2.35) yields

$$
-\operatorname{Im} \frac{u_{j}^{\prime}(a, \sqrt{z})}{u_{j}(a, \sqrt{z})}=\operatorname{Im} z \frac{\int_{0}^{a}\left|u_{j}(x, \sqrt{z})\right|^{2} d x}{\left|u_{j}(a, \sqrt{z})\right|^{2}}, \quad \operatorname{Im} z \neq 0 .
$$

Thus,

$$
\operatorname{Im}\left(-\frac{u_{j}^{\prime}(a, \sqrt{z})}{u_{j}(a, \sqrt{z})}\right) \geq 0 \quad \text { for } \operatorname{Im} z>0
$$


and consequently

$$
\operatorname{Im} \frac{\varphi_{j}(z)}{\psi_{j}(z)}=\operatorname{Im}\left(-\frac{u_{j}^{\prime}(a, \sqrt{z})}{u_{j}(a, \sqrt{z})}-\frac{\beta}{3}\right) \geq 0 \quad \text { for } \operatorname{Im} z>0 .
$$

Also, according to Lemma 2.10 the zeros of $\varphi_{j}(z)$ and $\psi_{j}(z)$ are real and hence $\varphi_{j}(z) / \psi_{j}(z) \epsilon$ $\mathscr{L}(\mathbb{C} \backslash \mathbb{R})$. Therefore, $\varphi_{j}(z) / \psi_{j}(z) \in \mathcal{N}$. Now it follows from (2.31) and (2.39) that $\varphi(z) / \psi(z) \in$ $\mathscr{H}(\mathbb{C} \backslash \mathbb{R})$ and

$$
\operatorname{Im} \frac{\varphi(z)}{\psi(z)}=\sum_{j=1}^{3} \operatorname{Im} \frac{\varphi_{j}(z)}{\psi_{j}(z)} \geq 0 \quad \text { for } \operatorname{Im} z>0
$$

Consequently $\varphi(z) / \psi(z) \in \mathcal{N}$. Lemma 2.11 is proved.

Lemma 2.12. The functions $\varphi_{j}(z) / \psi_{j}(z), j=1,2,3$ and $\varphi(z) / \psi(z)$ are of the class $\mathcal{N}_{-}^{e p}$.

Proof. By virtue of the formulas (2.12) we get

$$
\begin{aligned}
& u_{j}(a, \sqrt{z})=\frac{e^{\sqrt{|z| a}}(1+o(1)), \quad z \longrightarrow-\infty, j=1,2,}{2 \sqrt{|z|}} \\
& u_{3}(a, \sqrt{z})=\frac{e^{\sqrt{|z| a}}}{2}(1+o(1)), \quad z \longrightarrow-\infty, \\
& u_{j}^{\prime}(a, \sqrt{z})=\frac{e^{\sqrt{|z| a}}}{2}(1+o(1)), \quad z \longrightarrow-\infty, j=1,2, \\
& u_{3}^{\prime}(a, \sqrt{z})=\frac{\sqrt{|z|} e^{\sqrt{|z| a}}}{2}(1+o(1)), \quad z \longrightarrow-\infty .
\end{aligned}
$$

Using these asymptotics we obtain from (2.27) and (2.28)

$$
\frac{\varphi_{j}(z)}{\psi_{j}(z)}=-\sqrt{|z|}(1+o(1)), \quad z \longrightarrow-\infty, j=1,2,3
$$

and consequently

$$
\lim _{z \rightarrow-\infty} \frac{\varphi_{j}(z)}{\psi_{j}(z)}=-\infty, \quad j=1,2,3
$$

It follows form Lemmas 2.10 and 2.11, and (2.43) that there exist real numbers $b_{j} \in \mathbb{R}, j=$ $1,2,3$ such that

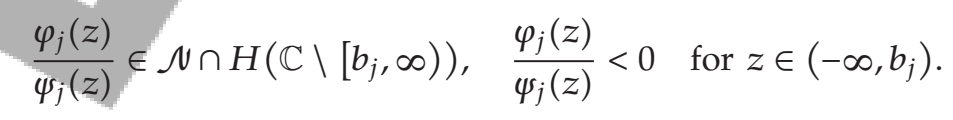

Therefore, $\varphi_{j}(z) / \psi_{j}(z) \in \mathcal{N}_{-}^{\mathrm{ep}}$ for $j=1,2,3$. 
Now using (2.31) and (2.44) and Lemma 2.11, we conclude that

$$
\frac{\varphi(z)}{\psi(z)} \in \mathcal{\mathcal { L }} \cap(\mathbb{C} \backslash[b, \infty)), \quad \frac{\varphi(z)}{\psi(z)}=\sum_{j=1}^{3} \frac{\varphi_{j}(z)}{\psi_{j}(z)}<0 \quad \text { for } z \in(-\infty, b),
$$

where $b=\min \left\{b_{1}, b_{2}, b_{3}\right\}$. Thus, $\varphi(z) / \psi(z) \in \mathcal{N}_{-}^{\mathrm{ep}}$. Lemma 2.12 is proved.

Theorem 2.13. The sequences $\left\{\lambda_{k}\right\}_{-\infty, k \neq 0}^{\infty}$ and $\left\{\kappa_{k}\right\}_{-\infty, k \neq 0}^{\infty}$ satisfy the following conditions:

(1) $0<\lambda_{1}<\kappa_{1} \leq \lambda_{2} \leq \kappa_{2} \leq \cdots \leq \lambda_{k} \leq \kappa_{k} \leq \cdots\left(\lambda_{-k}=-\lambda_{k}, \kappa_{-k}=-\kappa_{k}\right)$;

(2) $\kappa_{k}=\lambda_{k+1}$ if and only if $\lambda_{k+1}=\kappa_{k+1}$ for $k \in \mathbb{N}$;

(3) the maximal multiplicity of $\kappa_{k}$ is 3.

Proof. Denote $\mathcal{N}_{-}^{\mathrm{ep}}:=\mathcal{M}(\mathbb{C}) \cap \mathcal{N}_{-}^{\mathrm{ep}}$. The functions $u_{j}(a, \sqrt{z})$ and $u_{j}^{\prime}(a, \sqrt{z})$ are entire in $z$ and hence in view of Lemma 2.12, $\varphi_{j}(z) / \psi_{j}(z) \in \stackrel{\circ}{\text { ep }}$ for $j=1,2,3$ and $\varphi(z) / \psi(z) \in \mathcal{N}_{-}^{\text {ep }}$. Also, by Lemma 2.10 the functions $\varphi_{j}(z), \psi_{j}(z)$ have no common zeros. Therefore, the pairs $\left(\varphi_{j}, \psi_{j}\right), j=1,2,3$ are $1-\mathcal{N}_{-}^{\mathrm{ep}}$-pairs and consequently, in view of $(2.30)$, the pair $(\varphi, \psi)$ is an $m-\mathcal{N}_{-}^{\text {ep }}$-pair with some $m \leq 3$ (see Definition 2.6). On the other hand by virtue of (2.29), the squares of the zeros of $\Phi(\lambda)$ and $\Psi(\lambda)$ coincide with the zeros of $\varphi(z)$ and $\psi(z)$, respectively. Now the assertions of Theorem 2.13 immediately follows from [24, Corollary 4.6].

\section{Inverse Problem}

In the present section, we study the problem of reconstruction of the potential $q(x)=$ $\left[q_{j}(x)\right]_{j=1,2,3}$ and the real numbers $h, \beta$ from the given spectral characteristics. Let us denote by $Q$ the class of sets $\left\{\left[q_{j}(x)\right]_{j=1,2,3}, h, \beta\right\}$ which satisfy the following conditions:

(i) $q_{j}(x), j=1,2,3$ are real-valued functions from $L_{2}(0, a)$;

(ii) $h, \beta \in \mathbb{R}$;

(iii) the operator $A$ constructed via (1.6) is strictly positive.

Theorem 3.1. Let the following conditions be satisfied.

(1) Three sequences $\left\{v_{k}^{(j)}\right\}_{-\infty, k \neq 0}^{\infty}, j=1,2,3$ of real numbers are such that

(i) $v_{-k}^{(j)}=-v_{k}^{(j)}, v_{k}^{(j)}<v_{k+1}^{(j)}, v_{k}^{(j)} \neq 0$ for all $k \in \mathbb{N}$ and $j=1,2,3$;

(ii) $\left\{v_{k}^{(i)}\right\}_{-\infty, k \neq 0}^{\infty} \bigcap\left\{v_{k}^{(j)}\right\}_{-\infty, k \neq 0}^{\infty}=\emptyset$ for $i \neq j, i, j=1,2,3$;

(iii) one has

$$
v_{k}^{(j)}=\frac{\pi k}{a}+\frac{B_{j}}{\pi k}+\frac{\delta_{k}^{(j)}}{k^{2}}, \quad j=1,2
$$




$$
v_{k}^{(3)}=\frac{\pi(k-(1 / 2))}{a}+\frac{B_{3}}{\pi(k-(1 / 2))}+\frac{\delta_{k}^{(3)}}{k^{2}}
$$

where $B_{j}$ are real constants, $B_{i} \neq B_{j}$ for $i \neq j$ and $\left\{\delta_{k}^{(j)}\right\}_{-\infty, k \neq 0}^{\infty} \in l_{2}$ for $j=1,2,3$.

(2) A sequence $\left\{\lambda_{k}\right\}_{-\infty, k \neq 0}^{\infty}$ of real numbers $\left(\lambda_{-k}=-\lambda_{k}, \lambda_{k} \leq \lambda_{k+1}, \lambda_{k} \neq 0\right.$ for all $\left.k \in \mathbb{N}\right)$ can be represented as the union of three pairwise disjoint subsequences $\left\{\lambda_{k}\right\}_{-\infty, k \neq 0}^{\infty}=$ $\bigcup_{j=1}^{3}\left\{\lambda_{k}^{(j)}\right\}_{-\infty, k \neq 0}^{\infty}\left(\lambda_{-k}^{(1)}=-\lambda_{k}^{(1)}, \lambda_{-k}^{(2)}=-\lambda_{k}^{(3)}, \lambda_{-k}^{(3)}=-\lambda_{k}^{(2)}\right.$ and $\lambda_{k}^{(j)} \leq \lambda_{k+1}^{(j)}$ for $\left.j=1,2,3\right)$ which behave asymptotically as follows:

$$
\begin{gathered}
\lambda_{k}^{(1)}=\frac{k \pi}{a}+\frac{B_{1}+B_{2}}{2 k \pi}+\frac{r_{k}^{(1)}}{k^{2}}, \\
\lambda_{k}^{(j)}=\frac{k \pi+(-1)^{j} \sin ^{-1} \sqrt{2 / 3}}{a}+\frac{B_{0}}{k \pi}+\frac{r_{k}^{(j)}}{k^{2}}, \quad j=2,3,
\end{gathered}
$$

where $B_{0}$ is a real constant and $\left\{\gamma_{k}^{(j)}\right\}_{-\infty, k \neq 0}^{\infty} \in l_{2}$ for $j=1,2,3$.

(3) The sequences $\left\{\lambda_{k}\right\}_{-\infty, k \neq 0}^{\infty}$ and $\left\{\kappa_{k}\right\}_{-\infty}^{\infty}:=\bigcup_{j=1}^{3}\left\{\nu_{k}^{(j)}\right\}_{-\infty, k \neq 0}^{\infty} \bigcup\{0\}\left(\kappa_{-k}=-\kappa_{k}, \kappa_{k}<\kappa_{k+1}\right)$ interlace in the following strict sense:

$$
\cdots<\kappa_{-2}<\lambda_{-2}<\kappa_{-1}<\lambda_{-1}<\kappa_{0}=0<\lambda_{1}<\kappa_{1}<\lambda_{2}<\kappa_{2}<\cdots .
$$

Then there exists a unique set $\left\{\left[q_{j}(x)\right]_{j=1,2,3}, h, \beta\right\} \in Q$ such that the sequence $\left\{\lambda_{k}\right\}_{-\infty, k \neq 0}^{\infty}$ coincide with the spectrum of the boundary-value problem $L_{0}$, where $\beta=6 B_{0}-(3 / 2) B_{1}-(3 / 2) B_{2}-3 B_{3}$, $h=B_{3}-(1 / 2) \int_{0}^{a} q_{3}(x) d x$ and the sequences $\left\{v_{k}^{(j)}\right\}_{-\infty, k \neq 0}^{\infty}, j=1,2,3$ coincides with the spectra of the boundary-value problems $L_{j}, j=1,2,3$, respectively.

Proof. Denote by

$$
\begin{gathered}
\left\{\rho_{k}^{(0)}\right\}_{-\infty, k \neq 0}^{\infty}:=\left\{\frac{\pi k-\xi}{a}\right\}_{-\infty, k \neq 0}^{\infty} \bigcup\left\{\frac{\pi k+\xi}{a}\right\}_{-\infty, k \neq 0}^{\infty}, \quad \xi:=\sin ^{-1} \sqrt{\frac{2}{3}} \\
\left\{\rho_{k}\right\}_{-\infty, k \neq 0}^{\infty}:=\left\{\lambda_{k}^{(2)}\right\}_{-\infty, k \neq 0}^{\infty} \bigcup\left\{\lambda_{k}^{(3)}\right\}_{-\infty, k \neq 0}^{\infty} .
\end{gathered}
$$

It is possible to enumerate $\left\{\rho_{k}^{(0)}\right\}_{-\infty, k \neq 0}^{\infty}$ and $\left\{\rho_{k}\right\}_{-\infty, k \neq 0}^{\infty}$ in the proper way $\left(\rho_{-k}^{(0)}=-\rho_{k}^{(0)}, \rho_{k}^{(0)}<\right.$ $\rho_{k+1}^{(0)}$ and $\rho_{-k}=-\rho_{k}, \rho_{k} \leq \rho_{k+1}$. Let us construct the following entire functions:

$$
\begin{aligned}
& u_{j}(\lambda)=a \prod_{1}^{\infty}\left(\frac{a^{2}}{\pi^{2} k^{2}}\left(v_{k}^{(j) 2}-\lambda^{2}\right)\right), \quad j=1,2, \\
& u_{3}(\lambda)=\prod_{1}^{\infty}\left(\frac{a^{2}}{\pi^{2}(k-(1 / 2))^{2}}\left(v_{k}^{(3) 2}-\lambda^{2}\right)\right),
\end{aligned}
$$


ISRN Applied Mathematics

$$
\begin{aligned}
& \phi_{1}(\lambda)=a \prod_{1}^{\infty}\left(\frac{a^{2}}{\pi^{2} k^{2}}\left(\lambda_{k}^{(1) 2}-\lambda^{2}\right)\right), \\
& \phi_{2}(\lambda)=2 \prod_{1}^{\infty}\left(\frac{1}{\rho_{k}^{(0) 2}}\left(\rho_{k}^{2}-\lambda^{2}\right)\right) .
\end{aligned}
$$

Using [27, Lemma 2.1], we obtain

$$
u_{j}(\lambda)=\frac{\sin \lambda a}{\lambda}-B_{j} \frac{\cos \lambda a}{\lambda^{2}}+F_{j} \frac{\sin \lambda a}{\lambda^{3}}+\frac{f_{j}(\lambda)}{\lambda^{3}}, \quad j=1,2,
$$

where $F_{j}, j=1,2$ are constants and $f_{j}(\lambda) \in L^{a}$ for $j=1$,2. In the same way as [27, Lemma 2.1] we can prove that

$$
\begin{aligned}
& u_{3}(\lambda)=\cos \lambda a+B_{3} \frac{\sin \lambda a}{\lambda}+F_{3} \frac{\cos \lambda a}{\lambda^{2}}+\frac{f_{3}(\lambda)}{\lambda^{2}}, \\
& \phi_{1}(\lambda)=\frac{\sin \lambda a}{\lambda}-\left(\frac{B_{1}+B_{2}}{2}\right) \frac{\cos \lambda a}{\lambda^{2}}+G_{1} \frac{\sin \lambda a}{\lambda^{3}}+\frac{g_{1}(\lambda)}{\lambda^{3}}, \\
& \phi_{2}(\lambda)=2-3 \sin ^{2} \lambda a+3 B_{0} \frac{\sin 2 \lambda a}{\lambda}+G_{2} \frac{2-3 \sin ^{2} \lambda a}{\lambda^{2}}+\frac{g_{2}(\lambda)}{\lambda^{2}},
\end{aligned}
$$

where $F_{3}, G_{j}, j=1,2$ are constants and $f_{3}(\lambda), g_{1}(\lambda) \in L^{a}$ and $g_{2}(\lambda) \in L^{2 a}$.

Let us set

$$
\begin{gathered}
X_{k}^{(j)}:=v_{k}^{(j)}\left(\frac{\phi_{1}\left(v_{k}^{(j)}\right) \phi_{2}\left(v_{k}^{(j)}\right)}{u_{i}\left(v_{k}^{(j)}\right) u_{3}\left(v_{k}^{(j)}\right)}-\cos v_{k}^{(j)} a-B_{j} \frac{\sin v_{k}^{(j)}}{v_{k}^{(j)}}\right), i, j=1,2, i \neq j, \\
X_{k}^{(3)}:=\left(\frac{\phi_{1}\left(v_{k}^{(3)}\right) \phi_{2}\left(v_{k}^{(3)}\right)}{u_{1}\left(v_{k}^{(3)}\right) u_{2}\left(v_{k}^{(3)}\right)}+v_{k}^{(3)} \sin v_{k}^{(3)} a-B_{3} \cos v_{k}^{(3)} a\right)
\end{gathered}
$$

where the numbers $B_{j}, j=1,2,3$ can be determined by

$$
\begin{aligned}
& B_{j}=\lim _{k \rightarrow \infty} k \pi\left(v_{k}^{(j)}-\frac{\pi k}{a}\right), \quad j=1,2, \\
& B_{3}=\lim _{k \rightarrow \infty} \pi\left(k-\frac{1}{2}\right)\left(v_{k}^{(3)}-\frac{\pi(k-(1 / 2))}{a}\right) .
\end{aligned}
$$

It is clear that $X_{-k}^{(j)}=-X_{k}^{(j)}$ for $j=1,2$ and $X_{-k}^{(3)}=X_{k}^{(3)}$. To complete the proof we need the following lemma. 
Lemma 3.2. One has

$$
\left\{X_{k}^{(j)}\right\}_{-\infty, k \neq 0}^{\infty} \in l_{2} \text { for } j=1,2,3
$$

Proof. Substituting (3.1) into (3.8) and (3.9), we obtain

$$
\begin{aligned}
& u_{2}\left(v_{k}^{(1)}\right)=(-1)^{k} \frac{a^{2}\left(B_{1}-B_{2}\right)}{\pi^{2} k^{2}}+\frac{\zeta_{k}^{(1)}}{k^{3}} \\
& u_{3}\left(v_{k}^{(1)}\right)=(-1)^{k}\left(1-\frac{a^{2} B_{1}^{2}}{2 \pi^{2} k^{2}}+\frac{a^{2} B_{1} B_{3}}{\pi^{2} k^{2}}+\frac{a^{2} F_{3}}{\pi^{2} k^{2}}\right)+\frac{\zeta_{k}^{(2)}}{k^{2}} \\
& \phi_{1}\left(v_{k}^{(1)}\right)=(-1)^{k} \frac{a^{2}\left(B_{1}-B_{2}\right)}{\pi^{2} k^{2}}+\frac{\zeta_{k}^{(3)}}{k^{3}} \\
& \phi_{2}\left(v_{k}^{(1)}\right)=2+\frac{\zeta_{k}^{(4)}}{k}
\end{aligned}
$$

where $\left\{\zeta_{k}^{(j)}\right\}_{-\infty, k \neq 0}^{\infty} \in l_{2}$ for $j=\overline{1,4}$. Also, using (3.1), we obtain the asymptotic relation

$$
\cos v_{k}^{(1)} a+B_{1} \frac{\sin v_{k}^{(1)}}{v_{k}^{(1)}}=(-1)^{k}\left(1+\frac{\eta_{k}}{k}\right)
$$

where $\left\{\eta_{k}\right\}_{-\infty, k \neq 0}^{\infty} \in l_{2}$. If we substitute (3.14) and (3.15) into (3.10), then we conclude that $\left\{X_{k}^{(1)}\right\}_{-\infty, k \neq 0}^{\infty} \in l_{2}$. Analogously we can show that $\left\{X_{k}^{(2)}\right\}_{-\infty, k \neq 0}^{\infty} \in l_{2}$. We show that $\left\{X_{k}^{(3)}\right\}_{-\infty, k \neq 0}^{\infty} \in l_{2}$. Let us substitute (3.2) into (3.8) and (3.9). We obtain

$$
\begin{aligned}
& u_{j}\left(v_{k}^{(3)}\right)=\frac{(-1)^{k+1}}{v_{k}^{(3)}}\left(1+\frac{\zeta_{k}^{\prime(j)}}{k}\right), \quad j=1,2, \\
& \phi_{1}\left(v_{k}^{(3)}\right)=\frac{(-1)^{k+1}}{v_{k}^{(3)}}\left(1+\frac{\zeta_{k}^{\prime(3)}}{k}\right), \\
& \phi_{2}\left(v_{k}^{(3)}\right)=-1+\frac{\zeta_{k}^{\prime(4)}}{k},
\end{aligned}
$$

where $\left\{\zeta_{k}^{\prime(j)}\right\}_{-\infty, k \neq 0}^{\infty} \in l_{2}$ for $j=\overline{1,4}$. Furthermore, taking into account (3.2), we have

$$
v_{k}^{(3)} \sin v_{k}^{(3)} a-B_{3} \cos v_{k}^{(3)} a=(-1)^{k+1} v_{k}^{(3)}\left(1+\frac{\eta_{k}^{\prime}}{k}\right)
$$

where $\left\{\eta_{k}^{\prime}\right\}_{-\infty, k \neq 0}^{\infty} \in l_{2}$. Using (3.16) and (3.17) in (3.11), the assertion of Lemma 3.2 for $j=3$ follows. 
Now since the functions $\lambda u_{j}(\lambda), j=1,2$ and $u_{3}(\lambda)$ are sine-type functions (see Definition 2.9) and by virtue of (3.1), (3.2), and (3.5), $\inf _{k \neq p}\left|v_{k}^{(j)}-v_{p}^{(j)}\right|>0$ for $j=1,2,3$ (and hence the zeros of $\lambda u_{j}(\lambda), j=1,2$ and $u_{3}(\lambda)$ are simple), the Lagrange interpolation series

$$
\begin{aligned}
& \lambda u_{j}(\lambda) \sum_{\substack{-\infty \\
k \neq 0}}^{\infty} \frac{X_{k}^{(j)}}{d \lambda u_{j}(\lambda) /\left.d \lambda\right|_{\lambda=v_{k}^{(j)}}\left(\lambda-v_{k}^{(j)}\right)}, \quad j=1,2 \\
& u_{3}(\lambda) \sum_{\substack{-\infty \\
k \neq 0}}^{\infty} \frac{X_{k}^{(3)}}{d u_{3}(\lambda) /\left.d \lambda\right|_{\lambda=v_{k}^{(3)}}\left(\lambda-v_{k}^{(3)}\right)},
\end{aligned}
$$

constructed on the basis of the sequences $\left\{X_{k}^{(j)}\right\}_{-\infty, k \neq 0}^{\infty}$, define functions $\varepsilon_{j}(\lambda) \in L^{a}, j=1,2,3$, respectively (see [28, Theorem $\mathrm{A}]$ ). Using these functions, we define the even entire functions

$$
\begin{aligned}
& v_{j}(\lambda)=\cos \lambda a+B_{j} \frac{\sin \lambda a}{\lambda}+\frac{\varepsilon_{j}(\lambda)}{\lambda}, \quad j=1,2, \\
& v_{3}(\lambda)=-\lambda \sin \lambda a+B_{3} \cos \lambda a+\varepsilon_{3}(\lambda) .
\end{aligned}
$$

It follows directly from (3.18) and (3.19) that $\varepsilon_{j}\left(v_{k}^{(j)}\right)=X_{k}^{(j)}$ for $j=1,2,3$ and hence

$$
\begin{aligned}
& v_{j}\left(v_{k}^{(j)}\right)=\frac{\phi_{1}\left(v_{k}^{(j)}\right) \phi_{2}\left(v_{k}^{(j)}\right)}{u_{i}\left(v_{k}^{(j)}\right) u_{3}\left(v_{k}^{(j)}\right)}, \quad i, j=1,2, i \neq j, \\
& v_{3}\left(v_{k}^{(3)}\right)=\frac{\phi_{1}\left(v_{k}^{(3)}\right) \phi_{2}\left(v_{k}^{(3)}\right)}{u_{1}\left(v_{k}^{(3)}\right) u_{2}\left(v_{k}^{(3)}\right)} .
\end{aligned}
$$

Let us denote by $\left\{\mu_{k}^{(j)}\right\}_{-\infty, k \neq 0}^{\infty}$ the set of zeros of the functions $v_{j}(\lambda), j=1,2,3$, respectively. These sets are symmetric with respect to the real axis and to the imaginary axis. Hence, we number the zeros in the proper way: $\mu_{-k}^{(j)}=-\mu_{k}^{(j)}, \operatorname{Re} \mu_{k}^{(j)} \leq \operatorname{Re} \mu_{k+1}^{(j)}$ for all $k \in \mathbb{N}$ and the multiplicities are taken into account (we will prove that all $\mu_{k}^{(j) 2}$ are real and all $\mu_{k}^{(j)}$ are simple except for $\mu_{1}^{(j)}$, if $\mu_{1}^{(j)}=\mu_{-1}^{(j)}=0$ ). It follows from (3.20) that

$$
\begin{gathered}
\mu_{k}^{(j)}=\frac{\pi(k-(1 / 2))}{a}+\frac{B_{j}}{\pi(k-(1 / 2))}+\frac{\theta_{k}^{(j)}}{k}, \quad j=1,2, \\
\mu_{k}^{(3)}=\frac{(k-1) \pi}{a}+\frac{B_{3}}{k \pi}+\frac{\theta_{k}^{(3)}}{k},
\end{gathered}
$$

where $\left\{\theta_{k}^{(j)}\right\}_{-\infty, k \neq 0}^{\infty} \in l_{2}$ for $j=1,2,3$. 
Proposition 3.3. The following inequalities are valid:

$$
\mu_{1}^{(j) 2}<v_{1}^{(j) 2}<\mu_{2}^{(j) 2}<v_{2}^{(j) 2}<\cdots, \quad j=1,2,3
$$

Proof. In the same way as proof of [16, Proposition 2.3], we can show that

$$
\begin{gathered}
(-1)^{k} \frac{\phi_{1}\left(v_{k}^{(j)}\right) \phi_{2}\left(v_{k}^{(j)}\right)}{u_{i}\left(v_{k}^{(j)}\right) u_{3}\left(v_{k}^{(j)}\right)}>0, \quad i, j=1,2, i \neq j, \\
(-1)^{k} \frac{\phi_{1}\left(v_{k}^{(3)}\right) \phi_{2}\left(v_{k}^{(3)}\right)}{u_{1}\left(v_{k}^{(3)}\right) u_{2}\left(v_{k}^{(3)}\right)}>0 .
\end{gathered}
$$

From these inequalities and (3.21), it follows that

$$
(-1)^{k} v_{j}\left(v_{k}^{(j)}\right)>0, \quad j=1,2,3
$$

Let $j \in\{1,2,3\}$ be fixed. It follows from (3.26) that between consecutive $v_{k}^{(j)}$ 's there is an odd number (with account of multiplicities) of $\mu_{k}^{(j)}$ s. Suppose that there are three or more of them between $v_{k}^{(j)}$ and $v_{k+1}^{(j)}$. Then comparing (3.22) and (3.23) with (3.1) and (3.2), we conclude that there are no $\mu_{p}^{(j)}$ s s between some $v_{k^{\prime}}^{(j)}$ and $v_{k^{\prime}+1}^{(j)}$, where $k \neq k^{\prime}$, a contradiction. Thus, $v_{1}^{(j) 2}<\mu_{2}^{(j) 2}<v_{2}^{(j) 2}<\cdots$. If $v_{j}(0)>0$, then $0<\mu_{1}^{(j)}<v_{1}^{(j)}$. If $v_{j}(0)=0$, then $\mu_{1}^{(j)}=0$. If $v_{j}(0)<0$, then $\mu_{1}^{(j)}$ is a pure imaginary number and hence $\mu_{1}^{(j) 2}<v_{1}^{(j) 2}$. Proposition 3.3 is proved.

Let $j \in\{1,2\}$. It follows from (3.24) and the asymptotic relations (3.1) and (3.22) that the sequences $\left\{v_{k}^{(j)}\right\}_{-\infty, k \neq 0}^{\infty}$ and $\left\{\mu_{k}^{(j)}\right\}_{-\infty, k \neq 0}^{\infty}$ satisfy the conditions of [20, Theorem 3.4.1]. Thus, there exists a unique real-valued function $q_{j}(x) \in L_{2}(0, a)$ such that $\left\{v_{k}^{(j)}\right\}_{-\infty, k \neq 0}^{\infty}$ and $\left\{\mu_{k}^{(j)}\right\}_{-\infty, k \neq 0}^{\infty}$ are the spectra of the boundary-value problems $L_{j}$ and $L_{j}^{\prime}$, respectively. An algorithm for the reconstruction of this potential $q_{j}(x)$ is as follows (see [20, Section 3.4]): without loss of generality, let us assume that $\mu_{1}^{(j) 2}>0$, otherwise we apply a shift. The function

$$
e_{j}(\lambda)=e^{-i \lambda a}\left(v_{j}(\lambda)+i \lambda u_{j}(\lambda)\right)
$$

is the so-called Jost function of the corresponding prolonged Sturm-Liouville problem on the semiaxis:

$$
-y_{j}^{\prime \prime}(x)+\tilde{q}_{j}(x) y_{j}(x)=\lambda^{2} y_{j}(x), \quad x \in[0, \infty), y_{j}(0)=0,
$$


where

$$
\tilde{q}_{j}(x)= \begin{cases}q_{j}(x) & \text { if } x \in[0, a] \\ 0 & \text { if } x \in(a, \infty)\end{cases}
$$

Then we construct the $S$-function of the problem on the semi-axis:

and the function

$$
S_{j}(\lambda)=\frac{e_{j}(\lambda)}{e_{j}(-\lambda)}
$$

Solving the Marchenko integral equation

$$
K_{j}(x, t)+F_{j}(x+t)+\int_{x}^{\infty} K_{j}(x, s) F_{j}(x+s) d s=0, \quad t>x,
$$

we find the unique solution $K_{j}(x, t)$ and

$$
q_{j}(x)=-2 \frac{d K_{j}(x, x)}{d x}, \quad x \in[0, a] .
$$

The two sequences $\left\{v_{k}^{(3)}\right\}_{-\infty, k \neq 0}^{\infty}$ and $\left\{\mu_{k}^{(3)}\right\}_{-\infty, k \neq 0}^{\infty}$ satisfy (due to (3.2), (3.23) and (3.24)) the conditions of [19, Theorem 1.5.4]. Thus, there exists a unique real-valued function $q_{3}(x) \in$ $L_{2}(0, a)$ and a unique real number $h$ such that $\left\{v_{k}^{(3)}\right\}_{-\infty, k \neq 0}^{\infty}$ and $\left\{\mu_{k}^{(3)}\right\}_{-\infty, k \neq 0}^{\infty}$ are the spectra of the boundary-value problems $L_{3}$ and $L_{3}^{\prime}$, respectively. Below we give the algorithm of recovering of $q_{3}(x)$ as it is described in [19, Section 1.5]. Calculate the so-called weight numbers $\left\{\alpha_{k}\right\}_{1}^{\infty}$ of the problem $L_{3}^{\prime}$ by

where $\dot{v}_{3}(\lambda)=(\mathrm{d} / d \lambda) v_{3}(\lambda)$. If $\mu_{1}^{(3)}=0$, then $\dot{v}_{3}\left(\mu_{1}^{(3)}\right)=0$ and we set $\alpha_{1}=(1 / 2) \ddot{v}_{3}(0) u_{3}(0)$ where $\ddot{v}(\lambda)=\left(d^{2} / d \lambda^{2}\right) v_{3}(\lambda)$. Construct the function

$$
\alpha_{k}=\frac{1}{2 \mu_{k}^{(3)}} \dot{v}_{3}\left(\mu_{k}^{(3)}\right) u_{3}\left(\mu_{k}^{(3)}\right)
$$

$$
F(x, t)=\sum_{k=1}^{\infty}\left(\frac{\cos \mu_{k}^{(3) 2} x \cos \mu_{k}^{(3) 2} t}{\alpha_{k}}-\frac{\cos (k-1) x \cos (k-1) t}{\alpha_{k}^{0}}\right)
$$


where

$$
\alpha_{k}^{0}= \begin{cases}\frac{a}{2}, & k>1 \\ a, & k=1\end{cases}
$$

Then using the unique solution of the Gel'fand-Levitan integral equation

$$
K_{3}(x, t)+F(x, t)+\int_{0}^{x} K_{3}(x, s) F(s, t) d s=0, \quad 0 \leq t \leq x \leq a,
$$

we find

$$
q_{3}(x)=2 \frac{d K_{3}(x, x)}{d x}, \quad h=K_{3}(0,0)=B_{3}-\frac{1}{2} \int_{0}^{a} q_{3}(x) d x .
$$

To find $\beta$, we compare (2.21) and (3.4) and set

$$
\beta=6 B_{0}-\frac{3}{2} B_{1}-\frac{3}{2} B_{2}-3 B_{3}
$$

where $B_{0}$ can be determined by

$$
B_{0}=\lim _{k \rightarrow \infty} k \pi\left(\lambda_{k}^{(2)}-\frac{k \pi+\sin ^{-1} \sqrt{2 / 3}}{a}\right) .
$$

Now we prove that the spectrum of the problem $L_{0}$ which is generated by the obtained $\left[q_{j}(x)\right]_{j=1,2,3}, h$ and $\beta$ coincides with $\left\{\lambda_{k}\right\}_{-\infty, k \neq 0}^{\infty}$. Due to [20, Theorem 3.4.1] and [19, Theorem 1.5.4], the spectra of the problems $L_{j}, j=1,2,3$ which are generated by the obtained $\left[q_{j}(x)\right]_{j=1,2,3}$ and $h$ coincide with $\left\{v_{k}^{(j)}\right\}_{-\infty, k \neq 0}^{\infty}, j=1,2,3$, respectively. The functions $u_{j}(a, \lambda)(j=1,2,3)$ where $u_{j}(x, \lambda)$ is the solution of $(1.1)$ with obtained $\left[q_{j}(x)\right]_{j=1,2,3}$ and $h$ which satisfy the initial conditions (1.3) coincide with $u_{j}(\lambda)$, since they have the same zeros and the same asymptotics. Also, since $u_{j}^{\prime}(a, \lambda)$ and $v_{j}(\lambda)(j=1,2,3)$ have the same asymptotics and according to [20, Theorem 3.4.1] and [19, Theorem 1.5.4] have the same zeros, hence they coincide. Thus, the values of the function $\Phi(\lambda)$ (defined by $(2.5)$ ) at $\lambda=v_{k}^{(j)}$ coincide with

$$
\phi_{1}\left(v_{k}^{(j)}\right) \phi_{2}\left(v_{k}^{(j)}\right)
$$

for all $k \in \mathbb{Z} \backslash\{0\}$ and all $j=1,2,3$, that is, with the corresponding values of the function $\phi_{1}(\lambda) \phi_{2}(\lambda)$. This implies that the entire function

$$
\Delta(\lambda):=\Phi(\lambda)-\phi_{1}(\lambda) \phi_{2}(\lambda)
$$


of exponential type $3 a$ can be represented as follows:

$$
\Delta(\lambda)=t(\lambda) \prod_{j=1}^{3} u_{j}(a, \lambda)
$$

where $t(\lambda)$ is an entire function. Using (2.10) and (3.9) we have

$$
\begin{aligned}
\Delta(\lambda)= & t(\lambda)\left(\frac{\sin ^{2} \lambda a \cos \lambda a}{\lambda^{2}}-\left(B_{1}+B_{2}\right) \frac{\cos ^{2} \lambda a \sin \lambda a}{\lambda^{3}}+B_{3} \frac{\sin ^{3} \lambda a}{\lambda^{3}}+\frac{\omega_{1}(\lambda)}{\lambda^{3}}\right), \\
\phi_{1}(\lambda) \phi_{2}(\lambda)= & \frac{2 \sin \lambda a-3 \sin ^{3} \lambda a}{\lambda}+\left(6 B_{0}+\frac{1}{2} B_{1}+\frac{1}{2} B_{2}\right) \frac{\sin ^{2} \lambda a \cos \lambda a}{\lambda^{2}} \\
& -\left(B_{1}+B_{2}\right) \frac{\cos ^{3} \lambda a}{\lambda^{2}}+E_{1}^{\prime} \frac{\sin ^{3} \lambda a}{\lambda^{3}}+E_{2}^{\prime} \frac{\cos ^{2} \lambda a \sin \lambda a}{\lambda^{3}}+\frac{\omega_{2}(\lambda)}{\lambda^{3}},
\end{aligned}
$$

where $E_{1}^{\prime}, E_{2}^{\prime}$ are constants and $\omega_{1}(\lambda), \omega_{2}(\lambda) \in L^{3 a}$. Substituting (2.23) and (3.44) into (3.42) and using (3.39), we obtain

$$
\begin{gathered}
t(\lambda)\left(\lambda \sin ^{2} \lambda a \cos \lambda a-\left(B_{1}+B_{2}\right) \cos ^{2} \lambda a \sin \lambda a+B_{3} \sin ^{3} \lambda a+\omega_{1}(\lambda)\right) \\
=\left(E_{1}-E_{1}^{\prime}\right) \sin ^{3} \lambda a+\left(E_{2}-E_{2}^{\prime}\right) \cos ^{2} \lambda a \sin \lambda a+\omega_{3}(\lambda),
\end{gathered}
$$

where $\omega_{3}(\lambda) \in L^{3 a}$. Since the functions $\sin ^{3} \lambda a, \cos ^{2} \lambda a \sin \lambda a, \omega_{1}(\lambda)$ and $\omega_{3}(\lambda)$ are bounded on the real axis, hence relation (3.45) implies that $t(\lambda) \equiv 0$ and from (3.42), it follows that $\Phi(\lambda)=\phi_{1}(\lambda) \phi_{2}(\lambda)$. Consequently, the sequence $\left\{\lambda_{k}\right\}_{-\infty, k \neq 0}^{\infty}$ coincides with the spectrum of the boundary-value problem $L_{0}$. The operator $A$ constructed by (1.6) using the obtained $\left[q_{j}(x)\right]_{j=1,2,3}, h$ and $\beta$ is strictly positive, because it is self-adjoint and its spectrum is positive. The uniqueness of the solution of the inverse problem follows from the fact that formulas (3.18) and (3.19) establishes one-to-one correspondence between $l_{2}$ and $L^{a}$ (see [28, Theorem A]). The proof of Theorem 3.1 is finished.

Remark 3.4. If condition 1(ii) of Theorem 3.1 fails, that is, the sets $\left\{v_{k}^{(j)}\right\}_{-\infty, k \neq 0}^{\infty}, j=1,2,3$ are not pairwise disjoint (consequently, the condition 3 fails too), either the uniqueness or the existence result of mentioned theorem can also fails, for the same reasons as in the case of three spectra (see $[27,29]$ ). If the sequences $\left\{\lambda_{k}\right\}_{-\infty, k \neq 0}^{\infty}$ and $\left\{\kappa_{k}\right\}_{-\infty}^{\infty}=\bigcup_{j=1}^{3}\left\{v_{k}^{(j)}\right\}_{-\infty, k \neq 0}^{\infty} \bigcup\{0\}$ are not pairwise disjoint and satisfy the statements of Theorem 2.13 , then the solution of the inverse problem exists but is not unique.

\section{Acknowledgment}

This research is done with financial support of Research Office of the University of Tabriz. 


\section{References}

[1] E. Montrol, "Quantum theory on a network," Journal of Mathematical Physics, vol. 11, pp. 635-648, 1970.

[2] M. D. Faddeev and B. S. Pavlov, "A model of free electrons and the scattering problem," Akademiya Nauk SSSR, vol. 55, no. 2, pp. 257-268, 1983.

[3] K. Ruedenberg and C. W. Scherr, "Free-electron network model for conjugated systems-I. Theory," Journal of Chemical Physics, vol. 21, pp. 1565-1581, 1953.

[4] V. Kostrykin and R. Schrader, "Kirchhoff's rule for quantum wires," Journal of Physics A, vol. 32, no. 4, pp. 595-630, 1999.

[5] P. Kuchment, "Differential and pseudo-differential operators on graphs as models of mesoscopic systems," in Proceedings of the Analysis and Applications (ISAAC '01), vol. 10, pp. 7-30, Kluwer Academic Publishers, Dordrecht, The Netherlands, 2003.

[6] P. Kuchment, "Graph models for waves in thin structures," Waves in Random Media, vol. 12, no. 4, pp. R1-R24, 2002.

[7] G. Berkolaiko, E. B. Bogomolny, and J. P. Keating, "Star graphs and Šeba billiards," Journal of Physics A, vol. 34, no. 3, pp. 335-350, 2001.

[8] S. Currie, Spectral theory of differential operators on graphs, Ph.D. thesis, University of the Witwatersrand, Johannesburg, South Africa, 2006.

[9] Y. V. Pokornyi and A. V. Borovskikh, "Differential equations on networks (geometric graphs)," Journal of Mathematical Sciences, vol. 119, no. 6, pp. 691-718, 2004.

[10] Y. V. Pokornyi and V. L. Pryadiev, "The qualitative Sturm-Liouville theory on spatial networks," Journal of Mathematical Sciences, vol. 119, no. 6, pp. 788-835, 2004.

[11] V. Adamyan, "Scattering matrices for microschemes," in Operator Theory and Complex Analysis, vol. 59, pp. 1-10, Birkhäuser, Basel, Switzerland, 1992.

[12] R. Carlson, "Hill's equation for a homogeneous tree," Electronic Journal of Differential Equations, vol. 23, pp. 1-30, 1997.

[13] N. I. Gerasimenko and B. S. Pavlov, "A scattering problem on noncompact graphs," Akademiya Nauk SSSR, vol. 74, no. 3, pp. 345-359, 1988.

[14] Y. B. Melnikov and B. S. Pavlov, "Two-body scattering on a graph and application to simple nanoelectronic devices," Journal of Mathematical Physics, vol. 36, no. 6, pp. 2813-2825, 1995.

[15] Y. Melnikov and B. Pavlov, "Scattering on graphs and one-dimensional approximations to $\mathrm{N}$ dimensional Schrödinger operators," Journal of Mathematical Physics, vol. 42, no. 3, pp. 1202-1228, 2001.

[16] V. Pivovarchik, "Inverse problem for the Sturm-Liouville equation on a simple graph," SIAM Journal on Mathematical Analysis, vol. 32, no. 4, pp. 801-819, 2000.

[17] V. Pivovarchik, "Inverse problem for the Sturm-Liouville equation on a star-shaped graph," Mathematische Nachrichten, vol. 280, no. 13-14, pp. 1595-1619, 2007.

[18] C. F. Yang, "Inverse spectral problems for the Sturm-Liouville operator on a d-star garph," Journal of Mathematical Analysis and Applications, vol. 365, no. 2, pp. 742-749, 2010.

[19] G. Freiling and V. Yurko, Inverse Sturm-Liouville Problems and Their Applications, Nova Science, Huntington, NY, USA, 2001.

[20] V. A. Marchenko, Sturm-Liouville Operators and Applications, vol. 22, Birkhäuser, Basel, Switzerland, 1986.

[21] V. Pivovarchik, "Direct and inverse three-point Sturm-Liouville problem with parameter-dependent boundary conditions," Asymptotic Analysis, vol. 26, no. 3-4, pp. 219-238, 2001.

[22] B. Y. Levin, Lectures on Entire Functions, vol. 150, American Mathematical Society, Providence, RI, USA, 1996.

[23] R. P. Boas,, Entire Functions, Academic Press, New York, NY, USA, 1954.

[24] V. Pivovarchik and H. Woracek, "Sums of Nevanlinna functions and differential equations on starshaped graphs," Operators and Matrices, vol. 3, no. 4, pp. 451-501, 2009.

[25] B. Ja. Levin and I. V. Ostrovskii, "Small perturbations of the set of roots of sine-type functions," Izvestiya Akademii Nauk SSSR, vol. 43, no. 1, pp. 87-110, 1979 (Russian), English translatin in Mathematics of the USSR-Izvestiya, vol. 14, no. 1, pp. 79-101, 1980.

[26] N. Naimark, Linear Differential Operators, Parts I and II, Frederick Ungar, New York, NY, USA, 1968.

[27] V. N. Pivovarchik, "An inverse Sturm-Liouville problem by three spectra," Integral Equations and Operator Theory, vol. 34, no. 2, pp. 234-243, 1999. 
[28] B. J. Levin and J. I. Ljubarskiŭ, "Interpolation by entire functions belonging to special classes and related expansions in series of exponentials," Izvestiya Akademii Nauk SSSR, vol. 39, no. 3, pp. 657702, 1975.

[29] F. Gesztesy and B. Simon, "On the determination of a potential from three spectra," in Differential Operators and Spectral Theory, vol. 189, pp. 85-92, American Mathematical Society, Providence, RI, USA, 1999.
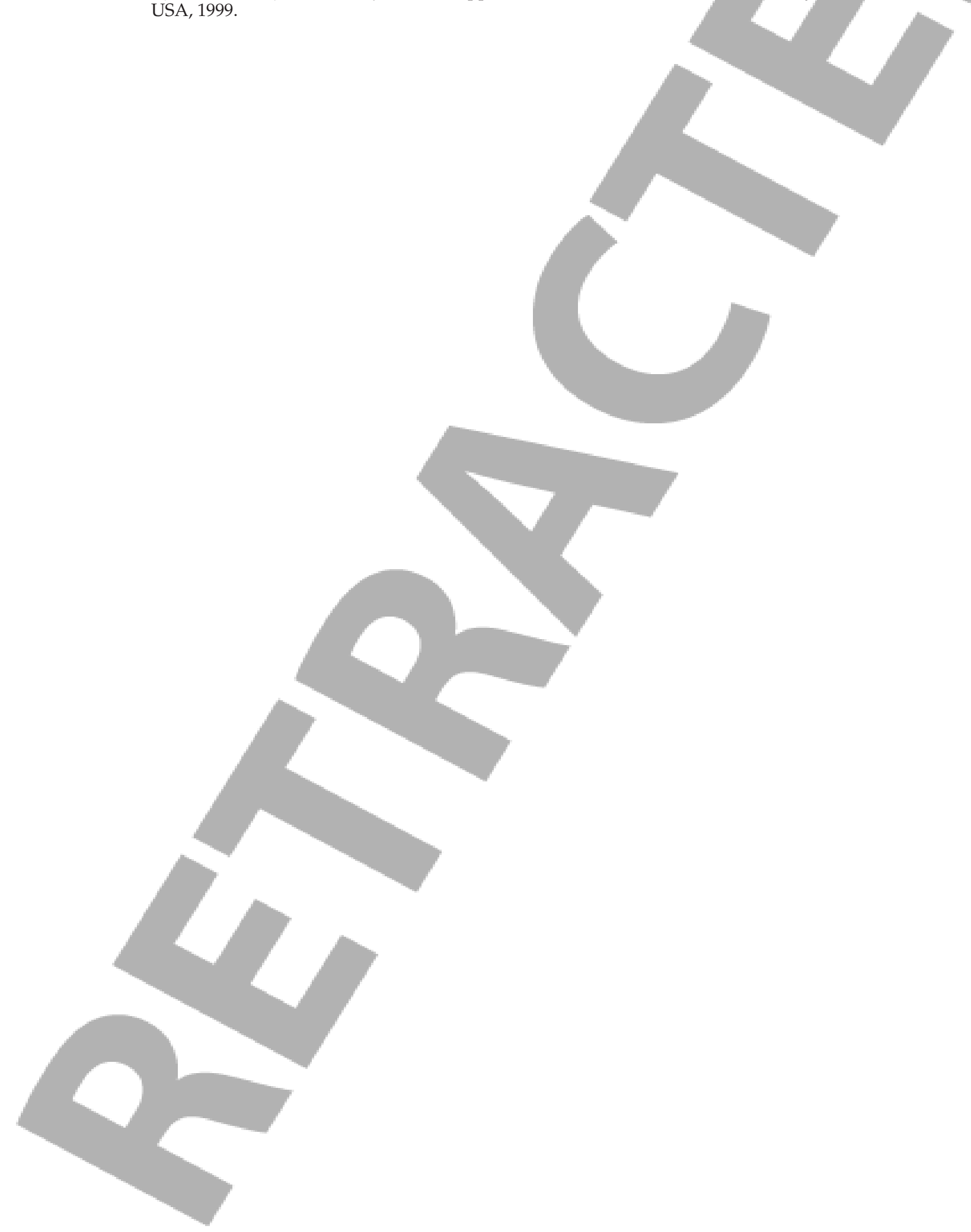